\title{
Anomalous Transverse Acoustic Phonon Broadening in the Relaxor Ferroelectric $\mathrm{Pb}\left(\mathrm{Mg}_{1 / 3} \mathrm{Nb}_{2 / 3}\right)_{0.8} \mathrm{Ti}_{0.2} \mathrm{O}_{3}$
}

\author{
T.-Y. Koo ${ }^{1}$, P. M. Gehring ${ }^{2}$, G. Shirane ${ }^{3}$, V. Kiryukhin ${ }^{1}$, G. Lee ${ }^{4}$, and S.-W. Cheong ${ }^{1,5}$ \\ ${ }^{1}$ Department of Physics and Astronomy, Rutgers University, Piscataway, New Jersey 08854 \\ ${ }^{2}$ NIST Center for Neutron Research, National Institute of Standards and Technology, Gaithersburg, Maryland 20899-8562 \\ ${ }^{3}$ Physics Department, Brookhaven National Laboratory, Upton, New York 11973 \\ ${ }^{4}$ iBLUe Photonics, Siheung-Si, Kyunggi-Do, Korea, 429-850 \\ ${ }^{5}$ Bell Laboratories, Lucent Technologies, Murray Hill, New Jersey, 07974
}

(October 26, 2018)

\begin{abstract}
The intrinsic linewidth $\Gamma_{T A}$ of the transverse acoustic (TA) phonon observed in the relaxor ferroelectric compound $\mathrm{Pb}\left(\mathrm{Mg}_{1 / 3} \mathrm{Nb}_{2 / 3}\right)_{0.8} \mathrm{Ti}_{0.2} \mathrm{O}_{3}(\mathrm{PMN}-20 \% \mathrm{PT})$ begins to broaden with decreasing temperature around $650 \mathrm{~K}$, nearly $300 \mathrm{~K}$ above the ferroelectric transition temperature $T_{c}(\sim 360 \mathrm{~K})$. We speculate that this anomalous behavior is directly related to the condensation of polarized, nanometer-sized, regions at the Burns temperature $T_{d}$. We also observe the "waterfall" anomaly previously seen in pure PMN, in which the transverse optic (TO) branch appears to drop precipitously into the TA branch at a finite momentum transfer $q_{w f} \sim 0.15 \AA^{-1}$. The waterfall feature is seen even at temperatures above $T_{d}$. This latter result suggests that the PNR exist as dynamic entities above $T_{d}$.
\end{abstract}

77.84.Dy, 63.20.Dj, 64.70.Kb, 77.80.Bh

\section{INTRODUCTION}

Relaxors are characterized by the ionic disorder at specific lattice sites. In the case of $\mathrm{Pb}\left(\mathrm{Mg}_{1 / 3} \mathrm{Nb}_{2 / 3}\right) \mathrm{O}_{3}$ $(\mathrm{PMN})$, considered by most researchers to be the prototype of the lead-oxide class of perovskite $\left(\mathrm{ABO}_{3}\right)$ relaxors, the disorder results from the random occupation of the B-site by two cations of differing valence, namely $\mathrm{Mg}^{2+}$ and $\mathrm{Nb}^{5+}$. Charge neutrality imposes the $\mathrm{Mg}: \mathrm{Nb}$ stoichiometry of $1 / 3: 2 / 3$, while the mixed-valence character of the B-site produces corresponding random electricfield gradients, and a locally broken translational symmetry. The associated dielectric susceptibilities exhibit unusually frequency-dependent maxima at a temperature $T=T_{\max }$ that are broad (diffuse) in temperature, and achieve very high values. Yet relaxors show no evidence of dong-range ferroelectric order when cooled in zero field. 1 Despite an intensive research effort spanning more than a decade, the physics of the "diffuse phase transition" is still controversial. 1 that such unusual properties are found in lead-oxide perovskite compounds such as PMN, which is a close relative of the prototypical displacive ferroelectric $\mathrm{PbTiO}_{3}$, is intrigung and suggests that the classic soft-mode description 5 of the phase transition, in which the lattice is unstable against the condensation of a zone-center transverse optic (TO) phonon, requires a conceptual extension in order to include the relaxor class of pseudo-ferroelectric systems. Indeed, the first definitive experimental evidence for a soft mode has been observed recently using neutron inelastic scattering techniques in PMN, but only at temperatures many hundreds of degrees above $T_{\max }(\approx 230 \mathrm{~K})$. For temperatures near or even a few hundred degrees above $T_{\text {max }}$, the soft mode is completely overdamped.
Single crystals of PMN and PZN $(Z=Z n)$ doped with $\mathrm{PbTiO}_{3}(\mathrm{PMN}-x \mathrm{PT}$ and $\mathrm{PZN}-x \mathrm{PT})$ at concentrations close to the rhombohedral side of the morphotropic phase boundary (MPB) display piezoelectric effects up to ten times greater than those of conventional ceramics which are based on PZT $\left(\mathrm{PbZr}_{1-x} \mathrm{Ti}_{x} \mathrm{O}_{3}\right)$ This remarkable finding by Park and Shrout in 1997 has affected work on industrial device applications as well as basic research on disordered dipolar systems. The subsequent, and unexpected, discovery by Noheda et al. 9 of a narrow region of monoclinic phase in PZT, sandwiched between the rhombohedral and tetragonal phases, sparked a new explanation of the origin of the exceptional piezoelectric

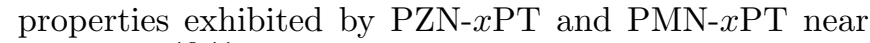
the MPB 10,11 As the techniques of synthesizing these materials have improved, large single crystals have become available, making feasible studies of the relaxor lattice dynamics using neutron inelastic scattering methods. One important finding of these studies was the so-called "waterfall" anomaly, first observed in measurements of the lowest-frequency TO phonons PZN-8\% PT at temperatures well above $T_{c}$ by Gehring et al.12 This feature is so named because the TO branch appears to dive into the TA branch at a specific reduced momentum transfer $q=q_{w f} \sim 0.20 \AA^{-1}$. This false impression results from a $q$-dependent damping of the TO mode that increases sharply at and below $q=q_{w f}$. The waterfall feature has since been observedin other systems such as PZN, PZN-15\%PT and PMN 13 It has been speculated that the polar nanoregions (PNR) that condense at a temperature $T_{d}$, several hundred degrees higher than either $T_{c}$ or $T_{\max }$, are the cause of the damping as their random polar nature should impede the propagation of the long-wavelength polar TO modes. The temperature $T_{d}$ 
was first identified by Burns and Dacol through measurements of the optic index of refraction of various relaxor and disordered ferroelectric systems, including PMN and PZN,14 and is known as the Burns temperature.

Motivated by these results, we have chosen to examine a high quality single crystal of PMN-20\%PT in which the relaxor behavior should be supressed relative to that observed in pure PMN, thus revealing the effects of ferroelectric $\mathrm{Ti}^{4+}$ on the waterfall anomaly. At this concentration a ferroelectric phase is stable in zero field (whereas it is not in PMN), the transition occurring at $T_{c}=360 \mathrm{~K}$. Since the the waterfall is thought to be induced by the PNR, its absence in PMN-20\%PT would imply an absence of the PNR (or at least a reduction in their density). Our results show, however, that even a $\mathrm{PbTiO}_{3}$ concentration of $20 \%$ is insufficient to erase the damping effects of the PNR on the TO mode. Instead, we observe an unusual broadening of the transverse acoustic (TA) phonon modes as well, the onset of which correlates well with the condensation of the PNR at the Burns temperature $T_{d}$. Interestingly, while the TO modes become highly overdamped below $T_{d}$ at low $q$, the TA modes remain well-defined. Nevertheless the damping effects on the TA modes are significant as the TA peak width in energy increases six-fold between $700 \mathrm{~K}$ and $400 \mathrm{~K}$.

\section{SAMPLE AND EXPERIMENTAL DETAILS}

A single crystal of PMN-20\%PT was grown using the modified Bridgeman method, and cut into a cube with dimensions $5 \times 5 \times 5 \mathrm{~mm}^{3}$. The room temperature lattice constant of PMN-20\%PT is $a=4.04 \AA$, thus 1 rlu (reciprocal lattice unit) corresponds to $2 \pi / a=1.555 \AA^{-1}$. The crystal was mounted on an aluminum sample holder during the first series of experiments, and then later remounted onto a boron nitride post using tantalum wire during subsequent experiments so as to avoid parasitic scattering from aluminum (which has nearly the same lattice constant as PMN). Each time the crystal was oriented with the cubic [010] axis vertical, thereby giving access to reflections of the form $(h 0 l)$. The sample was placed inside a closed-cycle helium refrigerator capable of reaching temperatures between $25 \mathrm{~K}$ and $670 \mathrm{~K}$. All of the neutron scattering data presented here were obtained on the BT9 triple-axis spectrometer located at the NIST Center for Neutron Research. The (002) reflection of highly-oriented pyrolytic graphite (HOPG) crystals was used to monochromate and analyze the incident and scattered neutron beams. An HOPG transmission filter was used to eliminate higher-order neutron wavelengths. Our data were taken holding the scattered neutron energy $E_{f}$ fixed at $14.7 \mathrm{meV}(\lambda=2.359 \AA)$ while varying the incident neutron energy $E_{i}$, and using horizontal beam collimations of 40'-46'-S-40'-80' ("S" = sample) from monochromator to detector.

The $\mathrm{PbTiO}_{3}$ (PT) content of our sample was con- firmed by measurements of both the dielectric susceptibility $(\epsilon)$ and the Bragg peak intensity as a function of temperature. The susceptibility at $10 \mathrm{kHz}$ exhibits a maximum at $38 \mathrm{~K}$, and is consistent with the published phase diagram 15 for this system. The temperature dependent scattering intensity of the (200) Bragg peak has also been used to measure the structural phase transition at $T_{c}$, which gives the PT concentration indirectly. In this study we define $T_{c}$ as $360 \mathrm{~K}$, where a rapid change in the (200) Bragg peak intensity (see the right inset of Fig. 2) takes place as a result of the relief of extinction due to the cubic-to-rhombohedral phase transition. The Burns temperature $T_{d}$ has not been identified for this sample. However, it can be roughly estimated assuming a linear extrapolation between the value of $T_{d}$ for PMN (about $620 \mathrm{~K}$ ) and the value of $T_{c}$ for $\mathrm{PbTiO}_{3}(763 \mathrm{~K})$, which gives $T_{d} \approx 650 \mathrm{~K}$.

\section{EXPERIMENTAL RESULTS}

\section{A. The waterfall anomaly}

Following the procedure outlined in prior studies of PZN-8\%PT, PZN, and PMN, we looked for the waterfall anomaly in PMN-20\%PT using standard constant$E$ scans in which the spectrometer energy transfer $E=$ $E_{i}-E_{f}$ is held fixed while scanning the momentum transfer $\vec{Q}=\overrightarrow{k_{i}}-\overrightarrow{k_{f}}$ along the crystalline cubic [001] direction. All such scans were measured in the (200) Brillouin zone at an energy transfer of $7 \mathrm{meV}$. In this manner the spectrometer sweeps out a horizontal path through $(E, \vec{Q})$ space that cuts through the region between the TO and TA branches as shown in the inset to Fig. 1. The results of constant- $E$ scans measured at $670 \mathrm{~K}, 500 \mathrm{~K}$, and $400 \mathrm{~K}$ are presented in Fig. 1, and give clear evidence of the waterfall in this system via the presence of the peak observed around $q=q_{w f} \approx 0.1 \AA^{-1}$, the intensity of which gradually diminishes as the temperature is lowered towards $T_{c}$. It is interesting to note that the waterfall is present even at temperatures slightly above $T_{d}$, as shown in top panel of Fig. 1.

As the temperature drops below $T_{d}$, the waterfall feature broadens in $q$ and finally smears out just above $T_{c}$. We note that the full width at half maximum (FWHM) of the waterfall peak, $\Delta q$, changes significantly between $500 \mathrm{~K}$ and $600 \mathrm{~K}$. At $670 \mathrm{~K}$ and $600 \mathrm{~K}$ (not shown in Fig. 1 for clarity) the FWHM $\Delta q \sim 0.12$ rlu, which increases to $0.16 \mathrm{rlu}$ at $500 \mathrm{~K}$. Thus a large change in the width takes place near $T_{d}$. As we will see in Fig.'s 3 and 4 , an anomaly over the same temperature range is also observed in constant- $\vec{Q}$ scans measured near the reciprocal lattice position of the waterfall, $\mathrm{q}_{w f}$. Interestingly, the scattering intensity measured at the zone center $(q=0)$ just above $T_{c}$ seems to increase as the temperature is lowered, and may be related to the recovery of the optic phonon mode at low temperature $(T=25 \mathrm{~K})$ observed in 
the PZN system 13 Below $T_{c}$, the crystal structure transforms from cubic to rhombohedral, so a direct comparison with the data above $T_{c}$ is not straightforward because the extinction effects in the cubic phase are released dramatically in the rhombohedral phase.

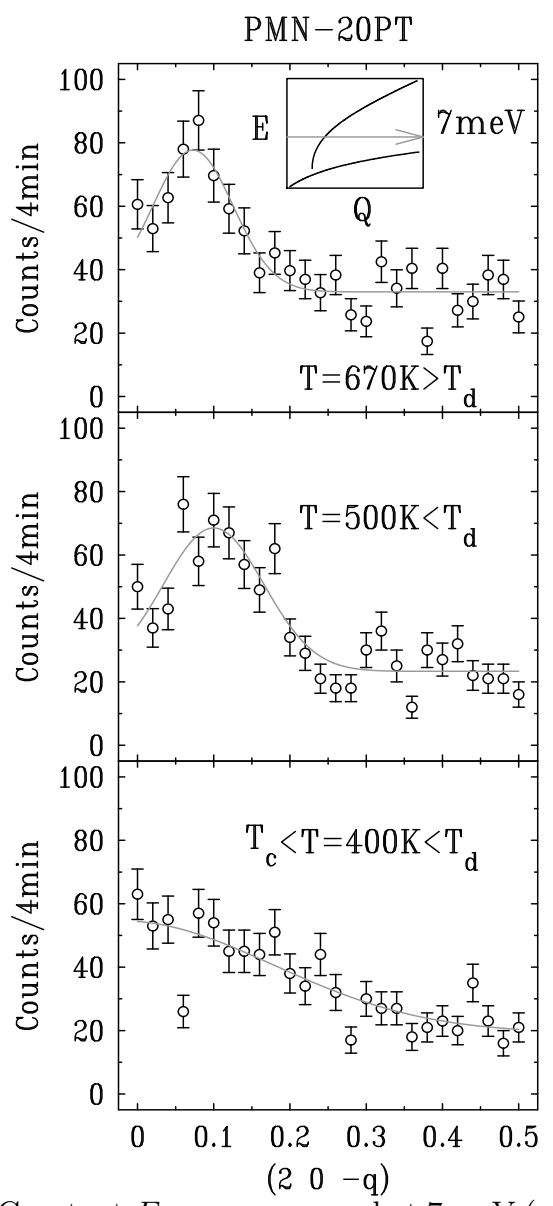

FIG. 1. Constant- $E$ scans measured at $7 \mathrm{meV}$ (phonon creation) for 670,500 , and $400 \mathrm{~K}$. The waterfall feature is seen clearly at $T=670 \mathrm{~K}$, close to $T_{d}(\sim 650 \mathrm{~K})$. The peak width increases, and the intensity diminishes, as the temperature is lowered, thereby indicating a change in the size/density of the PNR. The arrow in the inset shows the direction of scan.

\section{B. Diffuse scattering}

The waterfall anomaly occurs when the optic phonon modes near $q_{w f}$ become damped near $T_{d}$. This damping redistributes the TO mode neutron scattering cross section over a wide spectral range that overlaps with that of the acoustic modes. Depending on the extent of the damping, the TO mode cross section may even spill into the elastic channel. This latter possibility motivated our investigation of the diffuse scattering, as it could pick up contributions from a sufficiently broadened transverse optic phonon. During the course of these measurements, we noticed that the use of the aluminum sample holder gave rise to parasitic scattering that mimicked a spurious temperature-dependent diffuse scattering around (200). This false signal was generated not just because $\mathrm{Al}$ and PMN share similar lattice spacings (roughly $4.04 \AA$ ), but primarily because the thermal expansion of $\mathrm{Al}$ is such that the (200) powder line from the sample holder moves towards the PMN (200) peak near $300 \mathrm{~K}$ as shown by the broken line in Fig. 2. Generally $\mathrm{Al}$ is a preferred material for neutron experiments due to its comparatively high transparency with respect to other elements, and also because it is easily machined. However in this special case the presence of aluminum in the incident neutron beam gives rise to elastic scattering that is particularly insidious as it looks just like the signal one might expect due to a highly-damped TO phonon cross section. Therefore aluminum sample holders should not be used for measurements of the diffuse scattering in PMN. To avoid this problem we machined a flat, cylindrical sample holder made of strongly neutron-absorbing boron nitride for use in a subsequent set of measurements (using tantalum wire to hold the sample in place). These data are shown in Fig. 2.

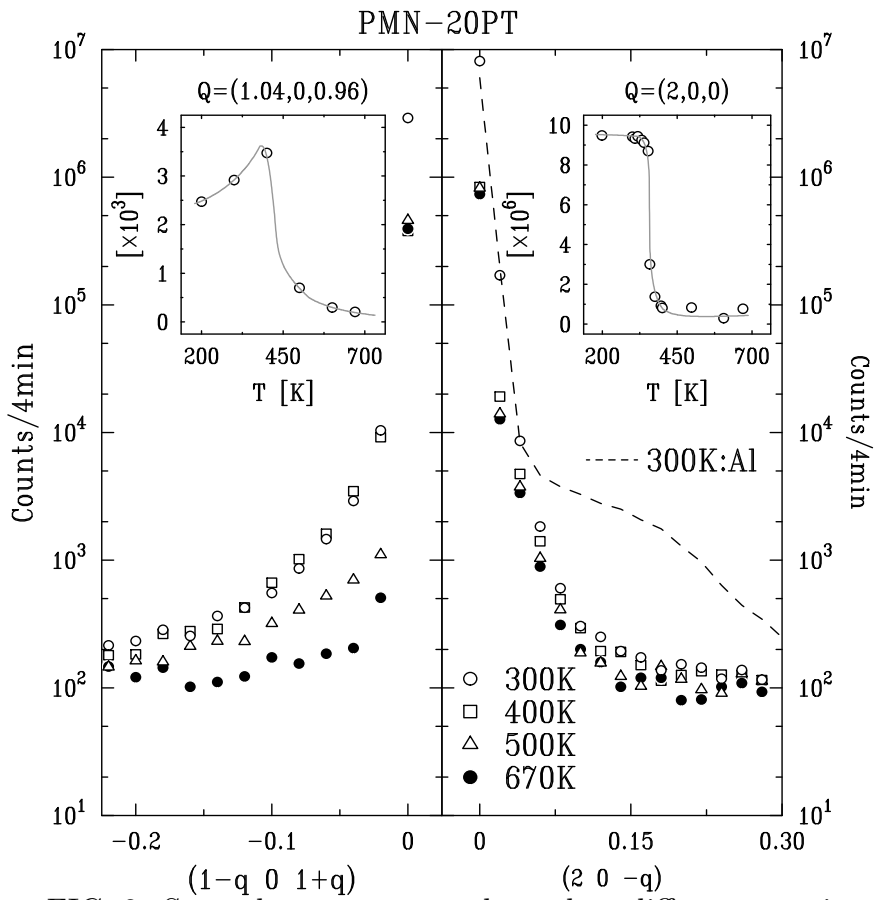

FIG. 2. Strongly temperature-dependent diffuse scattering is observed around the (101) Bragg peak. The temperature dependence of the diffuse intensity at $\vec{Q}=(1+h, 0,1-h)$, with $h=0.04$, is plotted at left. By contrast, almost no diffuse scattering is found near (200). $T_{c}$ is easily defined by the rapid change in intensity at (200). The broken line in the right panel represents the contamination from the $\mathrm{Al}$ sample holder used in an earlier set of measurements, which generates a spurious temperature-dependent diffuse scattering signature at (200).

Using the boron nitride sample holder, we find the surprising result that the diffuse scattering observed around (200), which is normally strong in perovskites, is extremely weak. Moreover, the diffuse scattering measured 
in the direction transverse to the scattering vector $\vec{Q}$ at (200) does not change much with temperature, as shown in the right panel of Fig. 2. However, a strong temperature dependence of the diffuse scattering is observed in the vicinity of the (101) Bragg peak. Similar results have been reported for the PZN system 16 The inset on the left side of Fig. 2 shows that the diffuse intensity at $\vec{Q}=(1.04,0,0.96)$ peaks near $T_{c} \sim 380 \mathrm{~K}$. This demonstrates the presence of critical scattering associated with normal ferroelectric fluctuations 17 in PMN-20\%PT, and stands in marked contrast to the case of pure PMN, for which the diffuse scattering intensity is reported to increase monotonically without any anomaly in the zerofield cooled state 18 But once an electric field is applied, which enhances the ferroelectric stability, the diffuse intensity diminishes appreciably, especially at low temperatures, becoming a broad maximum near the freezing temperature $T_{f}$. The observation of critical scattering around $T_{c}$ is direct evidence that PMN-20\%PT has lost some of its relaxor character, exhibiting instead classic ferroelectric behavior even in the absence of an external field, because the local random fields have been largely suppresed by the substitution with $\mathrm{Ti}^{4+}$ cations at the perovskite B-site.

\section{TA phonon damping}

Figure 3 shows data obtained from constant- $\vec{Q}$ scans measured at the scattering vector $\vec{Q}=(2,0,-0.12)$ near the waterfall anomaly at 670,500 , and $400 \mathrm{~K}$. The peak at $E=3 \mathrm{meV}$ is associated with the TA mode and is quite strong at high temperature. By contrast, the peak associated with the TO mode is difficult to locate because its spectral weight is so highly spread out in energy. Indeed, the absence of any distinct TO phonon peak indicates that these data were taken within the waterfall regime. The salient features of these scans can be understood using-mode coupling theory as described in earlier studies.13, 29 The most important feature of these data, however, is the abrupt increase of the half-width at half-maximum (HWHM) of the TA phonon peak, or the damping constant $\Gamma_{T A}$, near the Burns temperature $T_{d} \sim 650 \mathrm{~K}$. Naberezhnov et al. observed a strong temperature dependence to $\Gamma_{T A}$ in PMN at $(2,2,0.2)$ in which the TA mode starts to broaden at $T_{d}$.20 They further identified the approximate power law behavior $\Gamma_{T A} \propto q^{4}$ at $500 \mathrm{~K}$ over the range $0.03 \mathrm{rlu} \leq q \leq 0.07 \mathrm{rlu}$. This latter observation suggests that the TA damping effects are strongest near $q_{w f}$. We chose to study the TA damping for $q=0.12 \mathrm{rlu}$, which is only slightly above $q_{w f} \sim 0.10 \mathrm{rlu}$, for this reason.

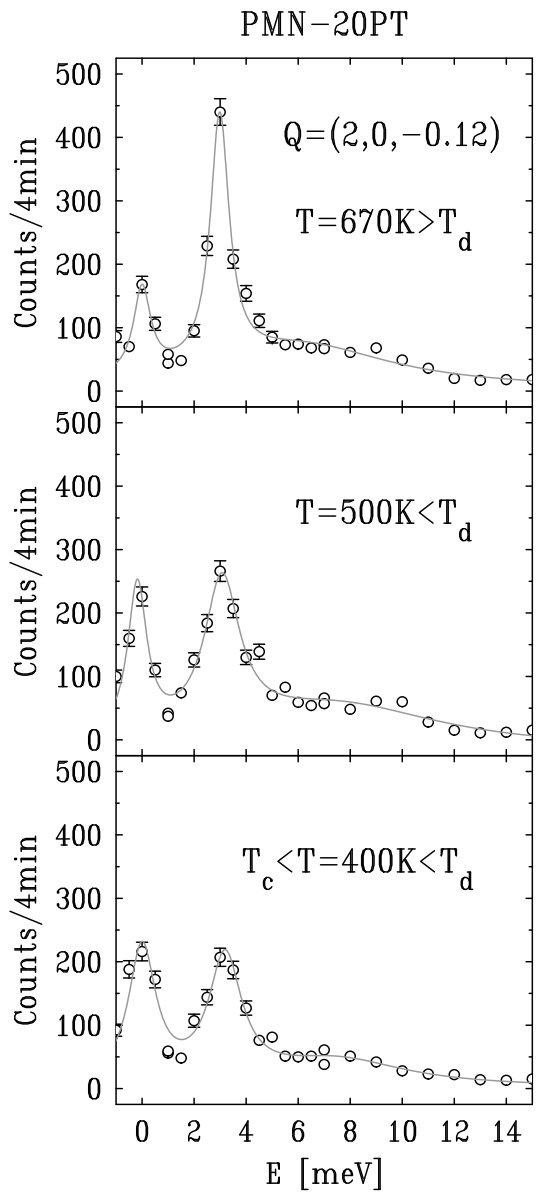

FIG. 3. Constant- $\vec{Q}$ scans measured at $(2,0,-0.12)$ for 670 , 500 , and $400 \mathrm{~K}$. Notice the increase of the TA phonon linewidth between $670 \mathrm{~K}$ and $500 \mathrm{~K}$.

To extract the intrinsic TA phonon HWHM $\Gamma_{T A}$, the data were fit to a Lorentzian function of $E$ and $g$ convolved with the experimental resolution function.21 The resulting values for $\Gamma_{T A}$ are plotted versus temperature in the bottom panel of Fig. 4. Notice that the intrinsic TA width begins to increase at a temperature very close to the extrapolated value for $T_{d}$ as indicated by the vertical dashed lines that extend down from the top panel. These data thus provide strong evidence that the PNR have a substantial damping effect on the TA modes as well as the TO modes, even in a PMN sample that contains a $20 \% \mathrm{Ti}^{4+}$ B-site occupancy. We note, however, that PMN-20\%PT still lies on the rhombohedral side of the MPB. Further experiments are required to determine whether or not the TA damping persists into the tetragonal side of the MPB, and if so, at what concentration it (and the waterfall anomaly) vanishes. 


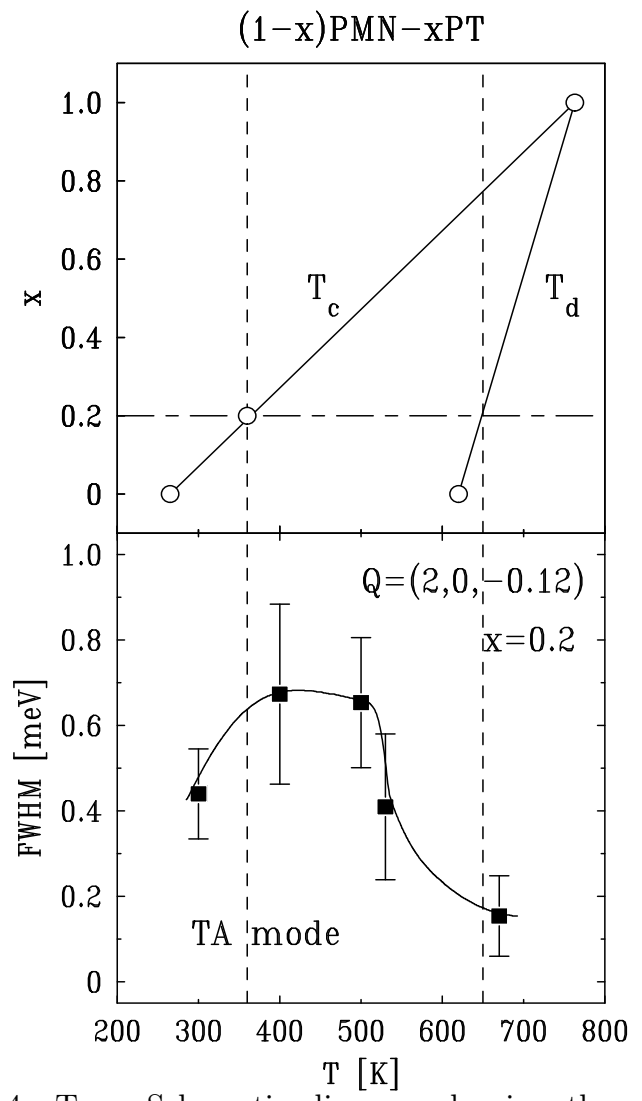

FIG. 4. Top: Schematic diagram showing the value of $T_{c}, T_{d}$ versus Ti concentration $x$ assuming a linear interpolation between $\mathrm{PMN}$ and $\mathrm{PbTiO}_{3}$. Bottom: Values for the intrinsic linewidths of the TA phonon peaks, shown in Fig. 3, after correcting for the instrumental resolution. The linewidth increases nearly sixfold between $700 \mathrm{~K}$ and $500 \mathrm{~K}$.

\section{DISCUSSION}

Chemical disorder is thought to inhibit the PNR from growing into regular micron-sized ferroelectric domains. The ordered cation domains in pure PMN are reported to be about $50 \AA$ in size, 22 whereas they are only $30 \AA$ in size in PMN-7\%PT 23 Synchrotron x-ray measurements on our PMN-20\%PT sample show no evidence of an Fspot (a Bragg reflection of the form $1 / 2(h, k, l)$ where $h, k, l$ are all integers) that would indicate the presence of short-range chemical order. Therefore the 20\% PT concentration is believed to be high enough to destroy the local nanometer-scale short-range 1:1 cation order. It is thus of great interest to study the resulting consequences on the PNR. This can be done by comparing the lattice dynamics of PMN-20\%PT with those of pure PMN since, as demonstrated by a series of neutron inelastic scattering studies of both PZN and PMN, the $q$-dependent damping of the polar $T$ phonon modes provides a sensitive probe of the PNR 12,13 that is unmatched by other spectroscopic techniques.

The extreme broadening of the TO modes produces the unusual waterfall feature, in which no clear optic phonon mode exists below a momentum transfer $q_{w f}$, as shown schematically in the inset to Fig. 1 . The constant- $E$ scans presented in the three panels of Fig. 1 reveal a peak in the scattering intensity similar to those observed in PMN, thereby indicating that the broadening also occurs in our PMN-20\%PT sample. More direct evidence of this is given in Fig. 3, where the TO mode manifests itself only as a weak shoulder around 7-9 meV. From this we can conclude that the PNR are still present in a sample of $\mathrm{PMN}-x \mathrm{PT}$ which has no local cation order. Whether or not the damping of the TO modes persists at higher $\mathrm{PbTiO}_{3}(\mathrm{PT})$ concentrations in the PMN-PT system is an important question because the substitution of $\mathrm{Ti}^{4+}$ for $\mathrm{Mg}^{2+} / \mathrm{Nb}^{5+}$ at the perovskite B-site changes the size of the cation short-range order (which limits the size of the PNR), as well as the random local field configuration (which affects the dielectric properties).

The constant- $\vec{Q}$ data in Fig. 3 further reveal an intriguing damping of the TA phonon modes, similar to that documented by Naberezhnov et al. in PMN. The TA damping is not as severe as that of the TO modes inasmuch as the TA modes remain well-defined at all temperatures. Nevertheless, the instrinsic TA phonon peak HWHM $\Gamma_{T A}$ measured near the waterfall wavevector $q_{w f}$ increases roughly sixfold between $700 \mathrm{~K}$ (just above $T_{d}$ ) and $400 \mathrm{~K}$ (just above $T_{c}$ ), as shown in Fig. 4 . It is interesting to note that the onset of the broadening of the TA modes appears to correlate more closely with $T_{d}$ than does the broadening of the TO modes at the same $q$ given that the waterfall anomaly is observed above $T_{d}$. This latter observation is extremely important, and it motivates our speculation that above $T_{d}$ the PNR exist as dynamic entities that impede the propagation of long-wavelength (low $q$ ) polar TO phonon modes. The zone center TO mode has been spown to soften as the temperature decreases towards $T_{d}$ while the increase in the diffuse scattering has been shown to correlate accurately with $T_{d} .20$ This suggests a picture in which the diffuse scattering results from critical ferroelectric fluctuations that condense into static regions of local polarization, i.e. PNR. Such critical fluctuations are evident in PMN-20\% PT as shown by the data plotted in the inset to Fig. 2, in which the near-Bragg scattering peaks at $T_{c} \sim 380 \mathrm{~K}$. In addition, the diffuse scattering further from the (101) Bragg peak shows no such anomaly at $T_{c}$, but instead starts to increase at a temperature consistent with our estimate of $T_{d} \sim 650 \mathrm{~K}$ for PMN20\%PT. Thus PMN-20\%PT exhibits both ferroelectric and relaxor character. After completing our measurements, Hirota et al. performed a detailed study of the diffuse scattering in pure PMN 24 the results of which are consistent with those discussed in this paper.

The behavior of the PNR is one of the most important issues in the study of relaxor ferroelectric systems. We have observed the waterfall anomaly over a wide temperature range, even above $T_{d}$, in a sample containing a relatively high concentration of ferroelectric $\mathrm{PbTiO}_{3}$. 
The characteristic peak widths in both $E$ and $q$ increase anomalously for the TA mode (E-scan) and the waterfall ( $q$-scan), respectively, near $T_{d}$. We speculate that these results are attributable to the nucleation of PNR around $T_{d}$. Similar studies on samples with different PT concentrations are now being planned.

\section{ACKNOWLEDGMENTS}

We would like to thank S. Wakimoto and Z.-G. Ye for stimulating discussions. This work was supported by the NSF under Grant No. DMR-9802513, and also Grant No. DMR-0093143. Work at Brookhaven National Laboratory was supported by the U. S. DOE under contract No. DE-AC02-98CH10886. We acknowledge the support of the NIST Center for Neutron Research, the U. S. Department of Commerce, for providing the neutron facilities used in the present work.

${ }^{1}$ L. E. Cross, Ferroelectrics 76, 241 (1987).

${ }^{2}$ V. Westphal, W. Kleemann, and M. D. Glinchuk, Phys. Rev. Lett. 68, 847 (1992).

${ }^{3}$ E. V. Colla, E. Yu. Koroleva, N. M. Okuneva, and S. B. Vakhrushev, Phys. Rev. Lett. 74, 1681 (1995).

${ }^{4}$ R. Blinc, J. Dolinsek, A. Grergorovic, B. Zalar, C. Filipic, Z. Kutnjak, A. Levstik, and R. Pirc, Phys. Rev. Lett. 83, 424 (1999).

${ }^{5}$ W. Cochran, Adv. Phys. 9, 387 (1960).

${ }^{6}$ P. M. Gehring, S. Wakimoto, Z. -G. Ye, and G. Shirane, cond-mat/0108170.

7 S.-E. Park and T. R. Shrout, J. Appl. Phys. 82, 1804 (1997).

${ }^{8}$ R. F. Service, Science 275, 1878 (1997).

${ }^{9}$ B. Noheda, D. E. Cox, G. Shirane, J. A. Gonzalo, L. E. Cross, and S.-E. Park, Appl. Phys. Lett. 74, 2059 (1999); B. Noheda, D. E. Cox, G. Shirane, R. Guo, B. Jones, and L. E. Cross, Phys. Rev. B 6301, 4103 (2001).

${ }^{10}$ H. Fu and R. E. Cohen, Nature 403, 281 (2000);D. Vanderbilt and M. H. Cohen, Phys. Rev. B 6309, 4108 (2001); L. Bellaiche, A. Garcia, and D. Vanderbilt, Phys. Rev. Lett. 84, 5427 (2000); L. Bellaiche and D. Vanderbilt, Phys. Rev. Lett. 83, 1347 (1999).

${ }^{11}$ B. Noheda, D. E. Cox, G. Shirane, S.-E. Park, L. E. Cross, and Z. Zhong, Phys. Rev. Lett. 86, 3891 (2001); D. E. Cox, B. Noheda, G. Shirane, Y. Uesu, K. Fujishiro, and Y. Yamada, Appl. Phys. Lett. 79, 400 (2001);

${ }^{12}$ P. M. Gehring, S.-E. Park, and G. Shirane, Phys. Rev. Lett. 84, 5216 (2000).

${ }^{13}$ P. M. Gehring, S. B. Vakhrushev, and G. Shirane, in Fundamental Physics of Ferroelectrics 2000, edited by R. E. Cohen (American Institute of Physics, Melville, NY, 2000), Vol. 535, p. 314; P. M. Gehring, S.-E. Park, and G. Shirane, Phys. Rev. B63, 224109 (2001); G. Shirane and P. M.
Gehring, J. Phys. Soc. Jpn. 70 Suppl. A, 227 (2001); D. La-Orauttapong, B. Noheda, Z.-G. Ye, P. M. Gehring, J. Toulouse, D. E. Cox, and G. Shirane, cond-mat/0108264.

${ }^{14}$ G. Burns and F. H. Dacol, Phys. Rev. B28, 2527 (1983); Solid State Commun. 48, 853 (1983).

15 J. Zhao, Q. M. Zhang, N. Kim and T. Shrout, Jpn. J. Appl. Phys. 34, 5658 (1995).

${ }^{16}$ D. La-Orauttapong, J. Toulouse, J. L. Robertson, and Z.G. Ye, cond-mat/0106051.

17 M. E. Lines and A. M. Glass, Principles and applications of ferroelectrics and related materials (Clarendon, Oxford, 1979).

${ }^{18}$ S. B. Vakhrushev, B. E. Kvyatkovsky, A. A. Naberezhnov, N. M. Okuneva, and B. P. Toperverg, Ferroelectrics 90, 173 (1989).

19 J. Harada, J. D. Axe, and G. Shirane, Phys. Rev. B 4, 155 (1971).

20 A. Naberezhnov, S. Vakhrushev, B. Dorner, D. Strauch, and H. Moudden, Eur. Phys. B11, 13 (1999).

${ }^{21}$ We used 3-axis resolution routines, ResLib programmed by Andrey Zheludev for this analysis.

${ }^{22}$ H. You and Q. M. Zhang, Phys. Rev. Lett. 79, 3950 (1997).

${ }^{23}$ A. D. Hilton, D. J. Barber, C. A. Randall, and T. R. Shrout, J. Mat. Sci. 25, 3461 (1990).

${ }^{24}$ K. Hirota, Z.-G. Ye, S. Wakimoto, P. M. Gehring, and G. Shirane, cond-mat/0109386. 